\title{
Dengue: A Review of the Laboratory Tests a Clinician Must Know to Achieve a Correct Diagnosis
}

\author{
Sérgio Oliveira De Paula ${ }^{1,2}$ and \\ Benedito Antônio Lopes da Fonseca ${ }^{1}$
}

\begin{abstract}
Department of Internal Medicine, School of Medicine of Ribeirão Preto $^{l}$; Program of Graduate Studies on Applied Microbiology and Immunology, School of Medicine of Ribeirão Preto - University of São Paulo², Ribeirão Preto/SP, Brazil
\end{abstract}

\begin{abstract}
Dengue is the most important disease caused by an arbovirus $(1,2,3$ and 4 serotypes) worldwide, especially in the tropical and sub-tropical regions. Its clinical manifestations range from asymptomatic infections to a severe disease characterized by hemorrhage and shock. The incidence of dengue virus activity in the Americas has substantially increased from 1980 to 1994. In Brazil, the increase in the incidence of dengue is especially linked to the dissemination of Aedes aegypti. Thus, a rapid and accurate dengue diagnosis is of paramount importance for effective control of dengue outbreaks [8]. Five serological tests have been used for the diagnosis of dengue infection: hemagglutination-inhibition (HI), complement fixation (CF), neutralization test (NT), immunoglobulin M (IgM) capture enzyme linked immunosorbent assay (MAC-ELISA) and indirect immunoglobulin G ELISA. The limitations of these techniques are the high cross-reactivity observed with these tests. Four methods of viral isolation have been routinely used for dengue viruses: intracerebral inoculation of newborn mice, inoculation on mammalian cell cultures, intrathoracic inoculation of adult mosquitoes, and inoculation on mosquito cell cultures. In recent years, several new diagnostic techniques have been developed and have proven very useful in dengue diagnosis, such as: nucleic and acid hybridization, RT-PCR. Currently, dengue diagnosis is based on serology, viral isolation and RNA detection. Enzyme-linked immunosorbent assays (ELISA) are still the most widely used technique for serological diagnosis, but they do not identify the dengue virus serotype responsible for the current infection, so molecular techniques may soon assume a very important role in dengue diagnosis. RT-PCR is definitely the most satisfactory test that can be used on these infections, since it has been shown to be able to detect dengue viruses up to the $10^{\text {th }}$ day after the onset of the symptoms.
\end{abstract}

Key Words: Dengue, diagnosis, serology, PCR.

Dengue results from infection by one of four dengue virus serotypes, named dengue-1, 2, 3, and 4; it is the most important disease caused by an arbovirus

Received on 17 August 2004; revised 11 November 2004. Address for correspondence: Dr. Benedito Antônio Lopes da Fonseca, M.D., Ph.D. - Laboratório de Virologia Molecular, Departamento de Clínica Médica, Faculdade de Medicina de Ribeirão Preto - Universidade de São Paulo. Avenida dos Bandeirantes, 3900. Ribeirão Preto - São Paulo, Zip code: 14049-900, Brazil. Fax: +55-16-633-0036/633-6695. E-mail: baldfons@fmrp.usp.br (Benedito A. L. Fonseca)

The Brazilian Journal of Infectious Diseases 2004;8(6):390-398 (C) 2004 by The Brazilian Journal of Infectious Diseases and Contexto Publishing. All rights reserved. worldwide, especially in the tropical and sub-tropical regions. Annually, dengue virus infections are responsible for up to 100 million cases of dengue fever and more than 500,000 cases of hemorrhagic fever $[1,2]$. Its clinical manifestations range from asymptomatic infections to a severe disease characterized by hemorrhage and shock, known as dengue hemorrhagic fever/dengue shock syndrome (DHF/DSS). DHF is characterized by plasma leakage and a hemorrhagic diathesis that is apparent by the time of defervescence, typically after five days of fever. In severe DHF, mortality results from hypotension and shock, at times accompanied by severe coagulation abnormalities and bleeding [3]. 
The factors responsible for the increasing importance of dengue as a global public health problem in the last decades are associated with demographic and social changes over the past 50 years. The major factors have been the unprecedented global population growth and the associated unplanned and uncontrolled urbanization, especially in tropical developing countries. Substandard housing, crowding, and deterioration in water, sewer and waste management systems, intimately associated with unplanned urbanization, have created ideal conditions for increased transmission of mosquito-borne diseases in tropical urban centers [4].

The incidence of dengue virus activity in the Americas has substantially increased from 1980 to 1994 . During this period, all dengue activity in the Americas was mainly associated with dengue serotypes 1,2 , and 4 (DEN-1, 2, and 4). The isolation of DEN-3 from two children hospitalized with minor hemorrhagic manifestations in Nicaragua, and from two persons with dengue fever in Panama at the end of 1994, marked the beginning of the detection of all four serotypes in the Americas [5].

In Brazil, the increase in the incidence of dengue is especially linked to the dissemination of Aedes aegypti. Dispersion of the vector was followed by the dissemination of serotypes 1 and 2 to most states of the country. DEN-3 was detected for the first time in December 2000 in the state of Rio de Janeiro, and subsequently, in the state of Roraima, in November $2001[6,7]$.

In order to prevent and control the progression of this disease, an expert committee of the World Health Organization proposed working guidelines that included the establishment of a surveillance system for dengue and DHF/DSS [4,8]. These guidelines rely on the precise and early diagnosis of dengue activity and reporting such activity to the public health authorities. Thus, a rapid and accurate dengue diagnosis is of paramount importance for effective control of dengue outbreaks [8].

Since dengue is a disease whose accurate diagnosis is difficult when based solely on clinical grounds, this article reviews the old and new laboratory techniques available for dengue diagnosis. These techniques were examined according to the type of the approach used for achieving the diagnosis, and the promising new molecular methods were examined in greater detail.

\section{Specific Laboratory Diagnosis}

The definitive diagnosis of dengue virus infection can only be made in the laboratory, and it depends on the isolation of these viruses, the detection of viral antigens or RNA in serum or tissues, or the detection of specific antibodies in the patients'serum $[9,10]$.

\section{Serologic Diagnosis}

Five serological tests have been used for the diagnosis of dengue infection: hemagglutinationinhibition ( $\mathrm{HI})$, complement fixation $(\mathrm{CF})$, neutralization test (NT), immunoglobulin M (IgM) capture enzyme linked immunosorbent assay (MAC-ELISA) and indirect immunoglobulin GELISA. The limitations of these techniques are the high cross-reactivity observed with these tests, requiring a comprehensive pool of antigens, including all four serotypes, another flavivirus (yellow fever virus, Japanese encephalitis virus, or St. Louis encephalitis virus), and in some areas, another virus that causes similar clinical manifestations but that is not flavivirus, such as Oropouche, Mayaro or Chikungunya viruses. Furthermore, the dengue antibodies are better detected around the fifth day of disease onset, making this technique unfeasible for rapid diagnosis [11].

\section{Hemagglutination-inhibition test (HI)}

For many years, the HI was the standard method used in dengue virus diagnosis due to its high degree of sensitivity and relatively easy execution. Denguespecific antibodies are detected for many years (48 years or more), being of great value for seroepidemiological studies and to differentiate primary from secondary infections. In primary infections, acute phase antibodies are detected from the fifth or sixth 
day of symptoms, usually when antibody titers are above 1:10. The antibody titers of convalescent phase samples are usually below 1:640 in primary infections. On the other hand, in secondary or tertiary infections denguespecific antibodies are readily detected, and there is a rapid increase of the titer during the first days of the infection, usually to a titer higher than 5:120. Thus, a titer of 1:1,280 or higher in samples collected during the acute phase or at the beginning of the convalescent phase of the disease is an indication of a dengue secondary infection. The highlevels of antibodies remain constant for two to three months in some patients when the titer of antibodies begins to fall. The main disadvantages of the HI test are its lack of specificity, the need for paired samples, and the inability to identify the infecting virus serotype [12].

\section{Complement fixation test $(C F)$}

The CF is usually not used for routine dengue diagnosis, since it is fairly difficulty to perform, requiring highly qualified and trained personnel to achieve good results. The test is based on the principle that the complement will be consumed during the antigenantibody reaction. The antibodies detected for $\mathrm{CF}$ generally appear later than $\mathrm{HI}$ antibodies and they persist for short periods, being of limited value for seroepidemiological studies. They are very specific in the primary infections, contributing to the determination of the infecting serotype, as demonstrated by the monotypic responses observed in primary infections [12].

\section{Neutralization test (NT)}

The NT is the most sensitive and specific serological test for dengue virus diagnosis, and it is detected for a long period of time. Due to its high specificity, NT can be used to identify the infecting serotype in primary dengue infections, since a relatively monotypic response is observed in the patients' serum during the convalescent phase. In secondary and tertiary infections, the determination of the infecting serotype by NT is not always reliable. The greatest disadvantages of this method are its high cost, the long time necessary to perform it, and the associated technical difficulties [11].

\section{Enzyme-linked immunosorbent assays (ELISAs)}

Up till now, ELISA has been considered the most useful test for dengue diagnosis, due to its high sensitivity and the ease of use. ELISA has been used to detect acute phase (IgM) and convalescent phase (IgG) antibodies, as well as for the detection of antigens (Ag). Since it is easy to perform and there is no need for sophisticated equipment, ELISA has become the most widely used serological method for dengue diagnosis. Also, due to its sensitivity for the detection of acute phase antibodies there is no need for convalescence samples [11-16] since anti-dengue IgM antibodies appear within five days of the the first clinical symptoms. The IgM production varies considerably among the patients. Some patients will have $\operatorname{IgM}$ detectable by the $2^{\text {nd }}$ to the $4^{\text {th }}$ day after the beginning of the symptoms, while others do not develop detectable IgM until the $8^{\text {th }}$ day after disease onset [12]. The IgM antibody titers in primary infections are significantly higher than in secondary infections, although the detection of titers of 1:320 in some cases is not uncommon [12]. The IgM production is much lower and transitory in secondary and tertiary infections [17]. A small percentage of patients have secondary infection with no IgM antibodies detected [17].

MAC-ELISA has been found to be much less sensitive than the HI test in paired serum samples collected during acute phase of the disease [12]. The specificity of MAC-ELISA is similar to that of $\mathrm{HI}$ in primary infections, as well as in secondary infections, when a monotypic response can be observed, but in general the response is crossreactive to all dengue serotypes and to other flaviviruses, such as those of Japanese encephalitis, St. Louis encephalitis and yellow fever viruses [12]. In dengue infections, the IgM monotypic response is not correlated with the serotype isolated from patients, and for this reason, MAC-ELISA cannot 
be used for viral identification [12]. There are several commercial kits available for dengue diagnosis by IgM-capture, including those made by well-known research institutes, such as the Oswaldo Cruz and Pedro Khouri Institutes.

MAC-ELISA has been a valuable tool for the surveillance of dengue and DHF/DSS. During epidemics, MAC-ELISA has the advantage of fast detection of the propagation of transmission. In areas where dengue is endemic, MAC-ELISA can be used as a valuable tool in the evaluation of a great number of clinical samples, with relative ease.

IgG-ELISA has been developed and is comparable to the HI test in the sense that it can be used for the differentiation of primary and secondary infections by dengue [18]. The test is simple and easy to do, and it can be used in the analysis of a great number of samples. IgG-ELISA is not very specific, cross-reacting with other flaviviruses, and it is not useful for dengue serotype identification. However, this technique is as highly sensitive as the HI test [4], and it might be useful in seroepidemiological studies.

Several other ELISAs for the detection of total immunoglobulin of flavivirus have been described and used in seroepidemiological studies and in serologic diagnoses [14,18-25].

Several serological assays have been developed to detect anti-dengue virus antibodies, taking into consideration the time necessary to diagnosis, simplicity and specificity of each of them [10,26$31]$. Hemolysis in gel $[33,34]$, the hemoadsorption immunosorbent technique [29] and the staphylococcal agglutination-inhibition reaction $[28,29]$ are examples of techniques used in a few laboratories [10], but none of them have been regularly used in virology labs.

\section{Viral Isolation}

Four methods of viral isolation have been routinely used for dengue viruses: intracerebral inoculation of newborn mice, inoculation on mammalian cell cultures, intrathoracic inoculation of adult mosquitoes, and inoculation on mosquito cell cultures $[10,11]$.

\section{Intracerebral inoculation}

Although all the four serotypes were initially isolated by intracerebral inoculation of suckling mice [34-36], this technique has several disadvantages, including high cost, long time for isolation, and low sensitivity. These problems have prevented further recommendation of this methodology for viral isolation.

\section{Mammalian cell culture}

Mammalian cell culture presents the same disadvantages as intracerebral inoculation. In order to be isolated with this system, the viruses frequently require multiple passages before inducing cytophatic effects in the infected cells. Although many laboratories have used this method, its use is not currently recommended for routine dengue virus isolation [11].

\section{Mosquito inoculation}

Mosquito inoculation is the most sensitive but the least used method for dengue virus isolation [37]. Four mosquito species have been used: Aedes aegypti, A. albopictus, Toxorhynchities amboinensis and $T$. splendens. Mosquitoes of both sexes are susceptible. Dengue virus generally replicates to high titers $\left(10^{6}\right.$ to $10^{7} \mathrm{MID}_{50}$ ) in as little as four to five days, depending on the incubation temperature. The detection is made through an indirect immunofluorescence assay (IFA) of mosquito tissues, usually done on the brain or salivary glands. The disadvantages are the hard work required, the need for insectaries to produce large numbers of mosquitoes for inoculation, and the isolation precautions taken to avoid the release of infected mosquitoes $[38,39]$.

\section{Mosquito cell culture}

Mosquito cell culture is the most recent methodology developed for dengue virus isolation [40-42]. Three cell lines of comparable sensitivity are frequently used [43], but the most widely used is the C6/36 clone of A. albopictus cells [41]. The use of this cell lineage 
has provided a rapid, sensitive and economical method for dengue virus isolation. Dengue antigens can be detected in infected cell culture by IFA. This technique is less sensitive than the intrathoracic inoculation of adult mosquitoes, but due to its ability to process several samples at the same time, it has become the standard technique for dengue virus isolation [40].

Compared to the other techniques, the advantages of the mosquito cells are: 1) higher sensitivity than the vertebrate cell lines for the recovery of dengue viruses [44], 2) they are relatively easy to maintain and grow at room temperature [42-45], and 3) it is possible to maintain cultures for up to 14 days without changing the medium [10].

Although some reports describe a cytopathic effect (syncytium formation, presence of multinucleated giant cells) induced by all four serotypes of dengue virus, the cytophatic effect produced in mosquito cell culture by many dengue viruses is difficult to detect, and it can be variable [42]. The cytophatic effect is usually seen when these cells are cultured in tubes.

\section{Molecular Detection}

In recent years, several new diagnostic techniques have been developed and have proven very useful in dengue diagnosis. These methods may assume an important role in dengue diagnosis, since they are able to readily detect the dengue viruses during the acute phase of the disease, and sometimes during the convalescence phase. The various methods are discussed below.

\section{Nucleic acid hybridization}

Nucleic acid hybridization, using RNA extracted from either dengue virus-infected cell culture supernatants or pools of infected A. albopictus, hybridized either with biotinylated probes [46] or ${ }^{32} \mathrm{P}$ labelled probes, is used primarily in epidemiological studies. It can also be used for viral diagnosis in tissues obtained in autopsies. The detection method using biotinylated probes is less sensitive than that using radiolabelled probes, and it has not been used for direct viral identification in clinical samples [47]. RNA-RNA hybridization is a sensitive technique that can be applied either directly on fresh samples or on retrospective analyses of fixed samples [48]. Due to the difficulties in working with RNA, as experienced technicians are required to obtain reproducible results, this method has been more often used as a research tool than a routine diagnostic method $[49,50]$.

\section{Reverse transcription - Polymerase Chain Reaction (RT-PCR)}

RT-PCR has been developed for the diagnosis of several diseases, and during the last years it has been revolutionizing the laboratorial diagnosis of infectious diseases. This method is rapid, sensitive, simple, and if correctly standardized, it can be used for genome detection in human clinical samples, biopsies, autopsy tissues or mosquitoes [52].

A number of RT-PCR procedures that detect and identify dengue serotypes in clinical specimens have been reported [51-57]. These PCR methods vary somewhat in terms of the amplified gene regions of the genome, in the ways they detect RT-PCR products, and the virus typing methods.

According to the World Health Organization (WHO), PCR is a powerful method to be used for dengue diagnosis, but it still needs to be better standardized. Aiming at the improvement of the molecular diagnosis of dengue, we have compared three well-established methods of RNA extraction from serum of patients with clinical symptoms of dengue. These methods were based on the QIAamp ${ }^{\circledR}$ Viral RNA kit, the Chomczynski-Sacchi technique and TRIzol ${ }^{\circledR}$. The data acquired in this study indicated that the best method of RNA extraction from clinical samples to be used for dengue diagnosis by RT-PCR is the QIAamp® Viral RNA kit [58].

In another study, we evaluated the detection of dengue virus by RT-PCR in blood, serum, and buffy-coats of 75 IgM-positive serum samples. Out of the 75 samples, 17 were positive for dengue using RT-PCR, and among these samples, 3 were positive in the blood, 14 positive in the serum and 8 positive in the buffy-coat. These results 
indicated that serum is the best clinical sample for RTPCR amplification of dengue genomes [59].

Due to the low detection rate of dengue virus by IFA on C6/36-infected cells when IgM-positive samples are used, we decided to test the performance of this cell culture to detect dengue virus in association with RT-PCR, aiming at increasing the sensitivity of viral detection. We compared the efficiency of RT-PCR and IFA for dengue-1 virus detection after inoculation of C6/36 cells with samples obtained during the convalescence period of dengue infection. Out of 75 IgM-positive samples inoculated into C6/36 cells, two were positive by IFA, while 17 were positive by RTPCR. We also investigated the time necessary for viral detection by both methods, using a fixed dose of $1 \times 10^{4}$ virus particles/mL. RT-PCR and IFA detected dengue virus one day and four days after virus inoculation, respectively [60]. Taken together, these results highlight the importance of PCR for the detection of dengue viruses after sample inoculation in C6/36 cells.

The results of several studies suggest that RT-PCR depends on the region of the genome that is chosen to be amplified and on the primers used to achieve this goal. RT-PCR can be type-specific in terms of dengue virus detection, and the detection threshold is usually less than 100 PFUs for all the serotypes.

Lanciotti et al. developed a RT-PCR test using consensual primers, designed to amplify the $\mathrm{C}$ and prM genes of dengue viruses. Their PCR was used to amplify a 511bp fragment through a protocol consisting of a conventional RT-PCR, followed by a nested-PCR, using specific primers for each dengue virus serotype [51].

In Brazil, Figueiredo et al. developed a RT-PCR for identification of Brazilian flaviviruses. The genomes of 13 Brazilian flaviviruses, except Bussuquara virus, were amplified by RT-PCR, using universal primers. Analysis of the RT-PCR products gave reproducible results and three distinct amplicon patterns were observed, allowing for correct identification of dengue viruses, as well as the other flaviviruses [56].

Since the WHO still considers PCR an experimental technique for dengue diagnosis, we conducted a validation study of PCR-based diagnosis with clinical samples collected in a region of Brazil where dengue-
1 virus has been circulating at a low incidence rate. Viral detection by RT-PCR was evaluated using the sera of patients with clinical diagnosis of dengue, and the results were compared to those obtained by IgMcapture enzyme-linked immunosorbent assay and virus isolation with the same samples. Our results demonstrated that RT-PCR is far more sensitive than virus isolation for clinical samples, allowing for rapid detection of dengue infections [57].

A great problem for dengue diagnosis has been the etiological confirmation of fatal cases. Frequently, only a single serum sample is obtained, and the serological test is of limited value in these situations. In these cases, it is now possible to detect antigens in a great variety of fixed samples with the new immunohistochemical methods [61,62]. Although immunofluorescence tests have been used in the past, new methodologies consisting of monoclonal or polyclonal antibodies labeled with peroxidase/ alkaline-phosphatase enzymes have improved the diagnosis of fatal cases based on fixed or fresh autopsy tissues [38].

Another important characteristic of PCR is its ability to identify the dengue serotype responsible for the ongoing disease. RT-PCR and restriction enzyme digestion of amplified DNAs have been used in combination, aiming at the development of a fast and simple virus identification method [63-65]. We developed a nested-PCR, which was followed by restriction enzyme digestion of the amplicons, to differentiate dengue- 1 from dengue- 2 , since at the time of that study these two serotypes were the most prevalent ones in Brazil. The samples were submitted to a nested-PCR amplification, and the amplicons were digested with Kpn I. These results were compared to virus isolation in C6/36 cells and to those obtained by conventional PCR. The use of nestedPCR yielded a three to four-fold increase in the detection rate of dengue virus. All of the amplicons digested by Kpn I identified dengue-1 virus as the infecting strain. These results indicated that nestedPCR provides a high yield of dengue genome amplification, even in the presence of IgM antibodies, and that restriction enzyme digestion rapidly defines the circulating serotype [65]. 
In addition to specific amplification and restriction enzyme analyses, other studies have demonstrated that nucleotide sequencing of gene fragments amplified by RT-PCR can be used as a fast method of genetic classification of dengue virus serotypes [52,66,67].

\section{Conclusion}

Since dengue infections may present as a severe disease, characterized by hemorrhage and shock, it is important to make a rapid diagnosis and, if possible, to identify the serotype involved in the infections. Correct diagnosis and serotype identification assume a greater importance for the severe form of the disease, since there is no marker that predicts the progression from classic dengue fever to the severe forms of the disease. The WHO acknowledges this fact and incentivates research on the development of new methodologies for dengue diagnosis.

Currently, dengue diagnosis is based on serology, viral isolation and RNA detection. Enzyme-linked immunosorbent assays (ELISA) are still the most widely used technique for serological diagnosis, but they do not identify the dengue virus serotype responsible for the current infection. Viral isolation has been difficult due to the presence of virusantibody immunocomplexes in convalescent patients, although detection of dengue virus antigen in mosquitoes has been accomplished in these cases. Recently, RT-PCR has been applied to nucleic acid detection in sera, tissues, and mosquitoes, and a number of different protocols have been used. As demonstrated in this review, molecular methods, especially RT-PCR, can be a very useful strategy for dengue virus detection and identification. Physicians involved in the care of dengue patients must be aware of the advantages and disadvantages of each method in order to provide the best standard of care to their patients.

Based on the data reviewed here, MAC-ELISA is still the most widely used diagnostic test, and it should be included in any investigation of dengue infection. However, molecular techniques may soon assume a very important role in dengue diagnosis.
RT-PCR is definitely the most satisfactory test that can be used on these infections, since it has been shown to be able to detect dengue viruses up to the $10^{\text {th }}$ day after the onset of the symptoms, and it is the technique that will provide the physician with the most useful body of information about the infection.

\section{References}

1. Halstead S.B. Pathogenesis of dengue: challenges to molecular biology. Science 1988;239:476-81.

2. Pinheiro F.P., Corber S.J. Global situation of dengue and hemorrhagic fever, and its emergence in the Americas. WHO 1997;50:161-8.

3. Libraty D.H., Young P.R., Pickering D., et al. High circulating levels of the dengue virus nonstructural protein NS1 early in dengue illness correlate with the development of dengue hemorrhagic fever. J Infect Dis 2002; 186(8): 1165-1168.

4. Race M.W., Fortune R.A.J., Agostini C., Varma M.G.R. Isolation of dengue viruses in mosquito cell culture under field conditions. Lancet 1978;1:48-9.

5. Gubler D.J. Dengue and dengue hemorrhagic fever. Clin Microbiol Reviews 1998;11(3):480-96.

6. Dengue. Activity in the Americas - 1994. Morbidity and Mortality Weekly Report 1995;44(2).

7. Dengue in Brazil: current situation and prevention and control activities. Epidemiological Bulletin - PAHO 2002;23(1).

8. Miagostovich M.P., Santos F.B., Simone T.S., et al. Genetic characterization of dengue virus type 3 isolates in the state of Rio de Janeiro, 2001. Braz J Med Biol Res 2002;35(8):869-72.

9. Dengue hemorrhagic fever, diagnosis, treatment and control. World Health Organization. Geneva, Switzerland, 1998.

10. King A., Innis B.L., Caudle L. B-cells are the principal circulating mononuclear cells infected by dengue virus. Faseb J 1991;5a:9998.

11. Guzman M.G. and Kouri G. Advances in dengue diagnosis. Clin Diagn Lab Immnunol 1996;3:621-7.

12. Vordam V., Kuno G. Laboratory diagnosis of dengue virus infections. in DJ Guber and G Kuno (ed). Dengue and dengue hemorrhagic fever, cab international, London, United Kingdon, pp 313-34. 1997.

13. Gubler D.J., Sathe R. Laboratory diagnosis of dengue and dengue hemorrhagic fever. in a Homma and IF Cunha, Proceedings of the international symposium on yellow fever and dengue, pp 291-322, 1988. 
14. Burke D.S., Nisalak A., Ussery M.A. Antibody capture immunoassay detection of Japanese encephalitis virus immunoglobulin $\mathrm{M}$ and $\mathrm{G}$ antibodies in cerebrospinal fluid. J Clin. Microbiol 1982; 15:1034-42.

15. Innis B.L., Nisalak A., Nimmannitya S., et al. An enzymelinked immunosorbent assay to characterize dengue infections where dengue and japanese encephalitis cocirculate. Am J Trop Med Hyg 1989;4:418-27.

16. Kuno G., Gubler D.J., Oliver A. Use the original antigenic sin theory to determine the serotypes of previous dengue infections. Trans R Soc Trop Med Hyg 1993;87:103-5.

17. Lam S.K., Devi S., Pang T. Detection of specific IgM in dengue infections. Southeast Asian J Trop Med Public Health 1987; 18:532-8.

18. Kuno G., Gomez I., Gubler D.J. An ELISA procedure for the diagnosis of dengue infections. J Virol Methods 1991;33:101-13.

19. Chungue E., Marché R., Plichart R., et al. Comparison of immunoglobulin G enzyme-linked immunosorbent assay (IgG-ELISA) and hemagglutination inhibition (HI) test for the detection of dengue antibodies. Prevalence of dengue IgG-ELISA antibodies in Tahiti. Trans R Soc Trop Med Hyg 1989;83:708-11.

20. Dittmar D., Cleary J., Castro A. Immunoglobulin G- and Mspecific enzyme-linked immunosorbent assay for detection of dengue antibodies. J Clin Microbiol 1979;9:498-502.

21. Fernadéz R, Vásquez S. Serological diagnosis of dengue by an ELISA inhibition method (EIM). Mem Inst Oswaldo Cruz 1990;85:347-51.

22. Figueiredo L.T., Costa M., Baeta S.M. Enzyme immunoassay for the detection of dengue IgM and IgG antibodies using mosquito cells as antigen. Trans $\mathrm{R}$ Soc Trop Med Hyg 1989;83:702-7.

23. Figueiredo L.T., Shope R. An enzyme immunoassay for dengue antibody using infected cultured mosquito cells as antigen. J Virol Methods 1987; 17:191-8.

24. Vásquez S., De La Cruz F., Guzmán M.G., Fernández R. Comparación de la técnica de fijación del complemento, la inhibición de la hemaglutinación y el inmunoensayo enzimático sobre fase sólida, para el diagnóstico del dengue. Rev Cub Med Trop 1986;38:7-14.

25. Vásquez S., Fernández R. Utilización de un método de inhibición de ELISA en el diagnóstico serológico del dengue. Reporte Preliminar Rev Cub Med Trop 1989; $41: 18-26$.

26. Fonseca B.A.L., Khoshnood K., Shope R.E., Mason P.W. Flavivirus type-specific antigens produced from fusions of a part of the E protein gene with the Escherichia coli trpe gene. Am J Trop Med Hyg 1991;44(5):500-8.

27. Cardosa M.J., Phaik T., Sham N. Development of a Dot enzyme immunoassay for dengue 3 : a sensitive method for the detection of anti-dengue antibodies. J Virol Methods 1988;22:81-8.
28. Chan Y.C., Tan H.C., Tan S.H., Balachandran K. The use of the single radial haemolysis in the serological diagnosis of dengue and Japanese encephalitis virus infections. Bull WHO 1985; 63:1043-53.

29. Chan Y.C., Tech S.H. Staphylococcal agglutinationinhibition reaction: a rapid and simple test for dengue antibodies. Sing Med 1975; 16:194-5.

30. Gunasekaran K., Pang T., Ahmed A., et al. Hemadsorption immunosorbent technique for the detection of dengue immunoglobulin $\mathbf{M}$ antibody. J Clin Microbiol 1986;23:170-4.

31. Lim K.A., Pong W.S. Agglutination by antibody of erythrocytes sensitized by virus dengue haemagglutinin. J Immunol 1964;92:638-47.

32. Okuno Y.T., Fukunaga T., Srisupaluck S., Fukai K. A modified pap (peroxidase-anti-peroxidase) staining technique using sera from patients with dengue hemorrhagic fever (DHF): 4 step staining technique. Biken J 1979;22:131-5.

33. Guzman M.G., Vásquez S., Bravo J., Monteagudo R., Kourí G. Utilidad de la hemólisis radial para el diagnóstico del dengue. Rev Cub Med Trop 1985;37:238-45.

34. Thongcharoen P., Wasi C., Puthavathana P. Dengue viruses monograph on dengue /dengue hemorrhagic fever. ed., Prasert Thongcharoen. World Health Organization, New Delhi, India, 1993.

35. Hammon W.M., Rudnick A., Sather G. New hemorrhagic fevers of children in the Philippines and Thailand. Trans Assoc Am Physicians 1960; 73:140-55.

36. Hotta S., Kimura R. Experimental studies on dengue 1. Isolation identification and modification of the virus. $\mathrm{J}$ Infect Dis 1952;90:1-9.

37. Sabin A.B. Research on dengue during World War II. Am J Trop Med Hyg 1952; 1:30-50.

38. Rosen L, Gubler D.J. The use of mosquitoes to detect and propagate dengue viruses. Am J Trop Med Hyg 1974;21:1153-60.

39. Gubler D.J., Suharyono W., Sumarmo H.W., et al. Virological surveillance for dengue hemorrhagic fever in Indonesia using the mosquitoes inoculation technique. Bull WHO 1979;57:931-6.

40. Kuberski T.T., Rosen L. A simple technique for detection of dengue antigen in mosquitoes by immunoflurescence. Am J Trop Med Hyg 1977;26:533-7.

41. Gubler D.J., Kuno G., Sather G.E., et al. Use of mosquitoes cell culture and specific monoclonal antibodies for routine surveillance of dengue viruses. Am J Trop Med Hyg 1984;33:158-65.

42. Igarashi A. Isolation of Singh's Aedes albopictus cell clone sensitive to dengue and chikungunya viruses. J Gen Virol 1978;40:530-44.

43. Tesh R.B. A method for the isolation and identification of dengue viruses, using mosquito cell cultures. Am J Trop Med Hyg 1979;28:1053-9. 
44. Kuno G., Gubler D.G., Velez M., Oliver A. Comparative sensitivity of threemosquito cell lines for isolation of dengue viruses. Bull WHO 1985;63:279-86.

45. Race M.W., Willians R.A., Agostini C.F.M. Dengue in the caribbean: virus isolation in a mosquito (Aedes pseudoscutellaris) cell line. Trans R Soc Trop Med Hyg 1979;73:18-22.

46. Igarashi A. Isolation of Singh's Aedes albopictus cell clone sensitive to dengue and chikungunya viruses. J Gen Virol 1978;40:530-44.

47. Henchal E.A., Narupitis R., Feighny R., et al. Detection of dengue virus RNA using nucleic acid hybridization. J Virol Methods 1987;15:187-200.

48. Khan A.M., Wright P.J. Detection of flavivirus RNA in infected cells using photobiotin-labelled hybridization probes. J Virol Methods 1987;15:121-30.

49. Monath T.P., Ballinger M.E., Miller B.R., Salaun J.J. Detection of yellow fever viral RNA by nucleic acid hybridization and viral antigen by immunocytochemistry in fixed human liver. Am J Trop Med Hyg 1989;40:663-8.

50. Deubel V. The contribution of molecular techniques to the diagnosis of dengue infection. In DJ Gubler and G Kuno. Dengue and dengue hemorrhagic fever. CAB International, London, United Kingdom, pp 335-366, 1997.

51. Deubel V., Pierre V. Molecular techiniques for rapid and more sensitive detection and diagnosis of flavivirus, p. 227237. In R.C. Spencer, E.P. Wright, and S.W.B. Newsom (cd.), Rapid methods and automation in microbiology and immunology. Intercept, Andover, United Kingdom, 1994.

52. Deubel V., Laille M., Hugnot J.P., et al. Identification of dengue sequences by genomic amplification: rapid diagnosis of dengue virus serotypes in peripheral blood. J Virol Methods 1990;30:41-54.

53. Fulop L., Barrett D.T., Phillpotts R., et al. Rapid identification of flavivirus based on conserved NS5 gene sequences. J Virol Methods 1993;44:179-88.

54. Suk-Yin C., Kautner I., Sai-Kit L. Detection and serotyping of dengue viruses by PCR: a simple, rapid method for the isolation of viral RNA from infected mosquito larvae. South Asian J Trop Med Public Health 1994;25:258-61.

55. Tanaka M. Rapid identification of flavivirus using the polymerase chain reaction. J Virol Methods 1993;41:311-22.

56. Figueiredo L.T., Batista W.C., Kashima S., Nassar E. S. Identification of brazilian flaviviruses by a simplified reverse transcription-polymerase chain reaction method using flavivirus universal primers. Am J Trop Med Hyg 1998;59(3):357-62.

57. De Paula S.O., Pires Neto R.J., Corrêa J.A.C.,et al. The use of reverse transcription-polymerase chain reaction (RT-PCR) for the rapid detection and identification of dengue virus in an endemic region: a validation study. Trans R S Trop Med Hyg 2002;96:266-9.
58. De Paula S.O., Nunes C., Matos R., et al. Comparison of techniques for extracting viral RNA from isolationnegative serum for dengue diagnosis by polymerase chain reaction. J Virol Methods 2001;98(2):119-25.

59. De Paula S.O., Fonseca B.A.L. Optimizing dengue diagnosis by RT-PCR in IgM-positive samples: comparison of whole blood, buffy-coat and serum as clinical samples. J Virol methods 2002;102:113-7.

60. De Paula S.O., Lima D.M., Clotteau M., et al. Improved detection of dengue-1 virus from IGM-positive serum samples using C6/36 cell cultures in association with RT-PCR. Intervirology 2003;46(4):227-31.

61. Hall W.C., Crowell T.P., Watts D.M., et al. Demonstration of yellow fever and dengue antigens in formalin-fixed paraffin embedded human liver by immunohistochemical analysis. Am J Trop Med Hyg 1991;45:408-17.

62. Zaki S.R., Peters C.J. Viral hemorrhagic fevers, p. 347364. In D.H. Connor, F.W. Chandler, D.A. Schawartz, H. J. Manz, and E.E. Lack (ed.), Diagnostic pathology of infections diseases. Appleton \& lange, Stamford, Conn, 1997.

63. Vorndam V., Kuno G., Rosado N. A PCR-restriction enzyme technique for determining dengue virus subgroups within serotypes. J Virol Methods 1994;48:237-44.

64. Vorndam V., Nogueira R.M.R., Trent D.W. Restriction enzyme analysis of american region dengue viruses. Arch Virol 1994;136:191-6.

65. De Paula S.O., Malta D.L., Fonseca B.A.L. Detection and identification of dengue-1 virus from clinical samples by a nested-PCR followed by restriction enzyme digestion of amplicons. J Med Virol 2002;66(4):529-34.

66. Chow V.T.K., Seah C.L.K., Chan Y.C. Comparative analysis of NS3 sequences of temporally separated dengue 3 virus strains isolated from Southeast Asia. Intervirology 1994;37:252-8.

67. Deubel V., Nogueira R.M., Drouet M.T., et al. Direct sequencing of genomic cDNA fragments amplified by the polymerase chain reaction for molecular epidemiology of dengue-2 viruses. Arch Virol 1993; $129: 197-210$ 